\title{
PULMONARY VASCULAR PATTERNS IN PULMONARY HYPERTENSION
}

\author{
BY \\ A. E. DOYLE, J. F. GOODWIN, C. V. HARRISON, AND R. E. STEINER \\ From the Departments of Medicine, Morbid Anatomy, and Radiology, Postgraduate Medical \\ School of London and Hammersmith Hospital \\ Received October 1, 1956
}

The radiological appearances of the pulmonary vascular system in mitral stenosis with pulmonary hypertension have been reported previously (Goodwin et al., 1952; Davies et al., 1953). In mitral stenosis with much pulmonary hypertension arterial narrowing was demonstrated on plain X-ray films and on pulmonary arteriograms, in the lower zones and sometimes in the mid zones of the lungs.

This paper reports further studies of the pulmonary vascular pattern in mitral stenosis, and also in certain types of congenital heart disease with pulmonary hypertension.

\section{Methods and Selection of Patients}

Patients were studied by plain radiography of the chest, pulmonary arteriography or venous angiography, and cardiac catheterization. The radiographic material previously reported in mitral stenosis (Davies et al., 1953) has been used to compare the appearances with those of 20 cases with congenital heart disease in whom the pulmonary arterial systolic pressures were $70 \mathrm{~mm}$. $\mathrm{Hg}$ or more: all but 3 had right-to-left shunts. There were 8 patients with atrial septal defects, one patient with a single atrium, 5 patients with ventricular septal defects, one patient with atrial and ventricular septal defects and total anomalous pulmonary venous drainage, and 5 with patent ductus arteriosus, two of whom had reversed their flow. The chest radiographs were also examined of 15 patients with left-to-right shunts but pulmonary artery systolic pressures of less than $60 \mathrm{~mm}$. Hg.

In addition, 30 cases of mitral stenosis and 6 cases of congenital heart disease have been studied by postmortem pulmonary arteriography and by histological examination of the pulmonary arteries in various parts of the lung. The post-mortem pulmonary arteriograms were prepared by the following method.

The lungs were dissected out complete with their main arteries. After removing all post-mortem clot, the arteries were cannulated and all air bubbles removed under water. Formol-saline was then run through (about 1 litre of $15 \%$ per lung) to ensure satisfactory fixation. The lungs were then injected with a mixture of barium sulphate and gelatine usually at a pressure of $80 \mathrm{~mm}$. $\mathrm{Hg}$; if the pulmonary artery pressure in life was known to have been higher than this, they were injected at the known pressure. When injection was complete the cannulae were clamped and the lung distended by running formol-saline into the bronchi. The bronchi were not tied and the lungs were allowed to assume their normal size. They were then placed in iced water to set the gelatine and stored in formol-saline until fixation was complete (a minimum of 14 days). Details of the apparatus and the injection mass have been published elsewhere (Harrison and Wood, 1949). After radiographs of the whole lung had been taken, the lung was divided up into segments and further radiographs taken of the following segments: middle lobe or lingula, anterior upper lobe, apical upper lobe, apical lower lobe, posterior basal, anterior basal. These segments were subsequently cut in 5-mm. slices from hilum outwards at right angles to the main artery and histological sections taken at known levels.

In most cases both lungs were studied, but in some only one, the other being either unsuitable or kept for other purposes. No difference was found between right and left lungs in respect of their vessels. 
Control lungs were treated in a similar way, both sexes and various ages being examined. It was, therefore, possible to compare radiographs and histological sections with those of controls of roughly corresponding ages.

\section{(a) Mitral Stenosis}

\section{RADIOLOGICAL StUdies}

The cases reported here include those reported as Grade II pulmonary hypertension by Davies et al. (1953), the systolic pulmonary artery pressures being $70 \mathrm{~mm}$. of mercury or above. All patients had considerable dilatation of the main pulmonary arteries and main branches, and much narrowing of the smaller peripheral arteries in the lower and mid zones. Narrowing of the small peripheral arteries was never seen in the upper zones. Other changes were the occurrence of horizontal lines in the lower zones towards the periphery of the lung field in the costophrenic angles (Shanks and Kerley, 1951; Fleischner and Reiner, 1954). There was also a diffuse haziness in the lower zones. The appearances seen in the plain films were confirmed angiographically in those cases in which this investigation was performed (Fig. 1 and 2).

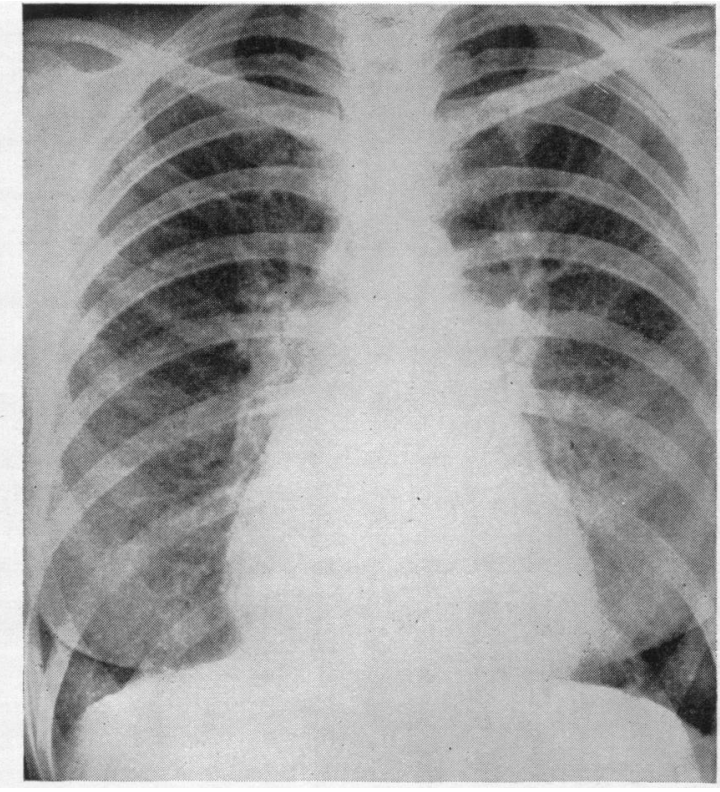

Fig. 1.-Severe pulmonary hypertension due to mitral stenosis. There is considerable enlargement of the main pulmonary arteries, with irregularity and narrowing of the peripheral arteries in the lower zones. The arteries to the upper zones, however, are not reduced in calibre. Faint costophrenic lines are also present.

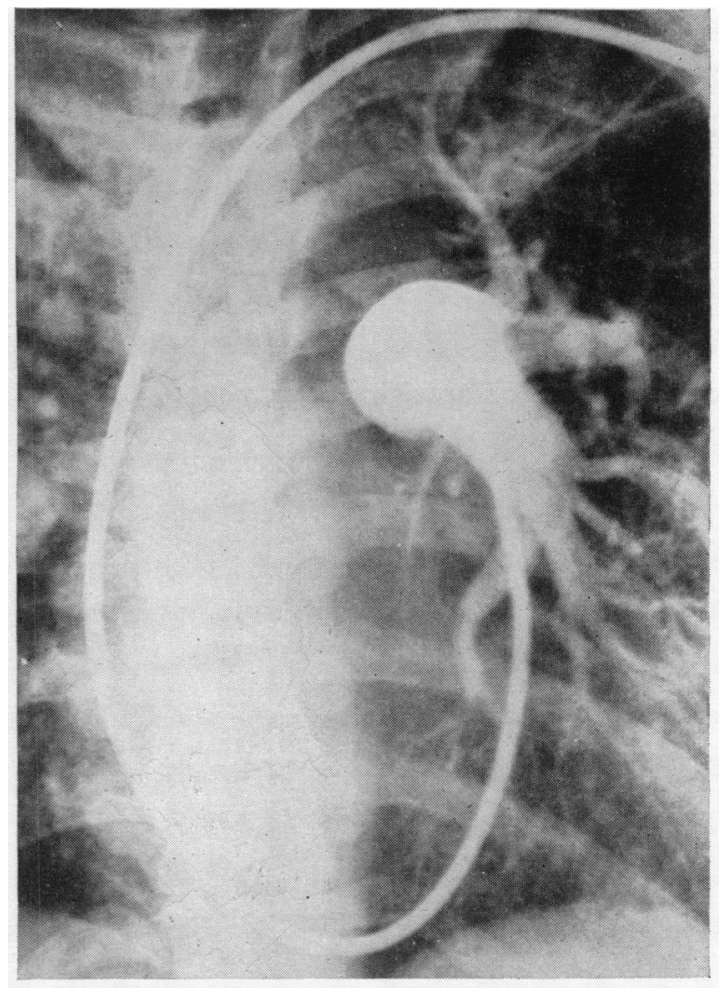

FIG. 2.-Left pulmonary arteriogram in severe pulmonary hypertension due to mitral stenosis. The left main pulmonary artery is greatly enlarged, but the medium-size branches to the lower zones are narrowed, in contrast to those in the upper zone.

\section{(b) Congenital Heart Disease with Pulmonary Hypertension}

The systolic pulmonary artery pressures ranged from 70 to $150 \mathrm{~mm}$. $\mathrm{Hg}$. Sixteen patients had some clinical cyanosis, due to reversal of the shunts. The following changes were noted in plain films and in angiocardiograms. 
As in the group with mitral stenosis, there was dilatation of the main pulmonary arteries in all patients. In two of these, both with reversed patent ductus arteriosus, the dilatation was moderate: in the remaining 18 , it was extreme.

In contrast to the findings in mitral stenosis, the medium-sized arteries both to the lower zones and the upper zones were larger than normal in 18 of the 20 patients studied. In the remaining 2 patients, one of whom had a ventricular septal defect, and the other, a patent ductus with totally reversed flow, the medium-sized branches were of normal size.

In 10 patients, the small peripheral arteries appeared to be of normal or larger than normal size and distribution. In the remaining 10 , the small peripheral arteries were narrower than normal or were not visible. As with the changes in the medium-sized arteries, the changes were evenly distributed throughout both upper and lower zones. There was no relationship between the peripheral arterial changes and the magnitude of the shunts present.

The costophrenic horizontal lines, which were frequently seen in mitral stenosis, were noted in only 3 of the 19 subjects studied. The diffuse haziness in the lower zones encountered in those with mitral stenosis, was not seen in any of the congenital group (Fig. 3, 4, 5, and 6).

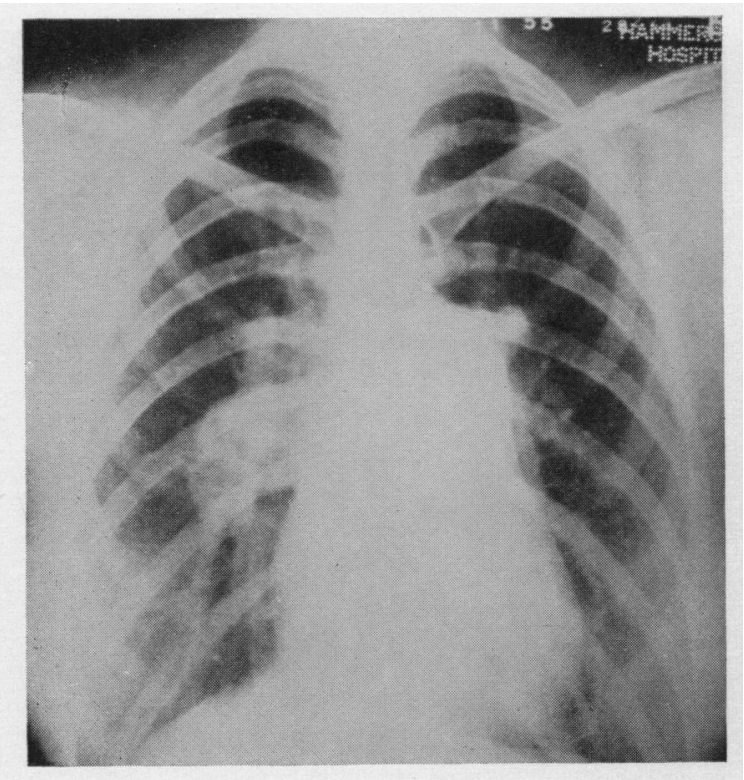

FIG. 3.-Atrial septal defect with bi-directional shunt and severe pulmonary hypertension. Shows enormous enlargement of main pulmonary artery and branches, and oligæmia of the peripheral lung fields present equally in upper, mid, and lower zones. The large pulmonary arteries showed marked intrinsic pulsation on fluoroscopy.

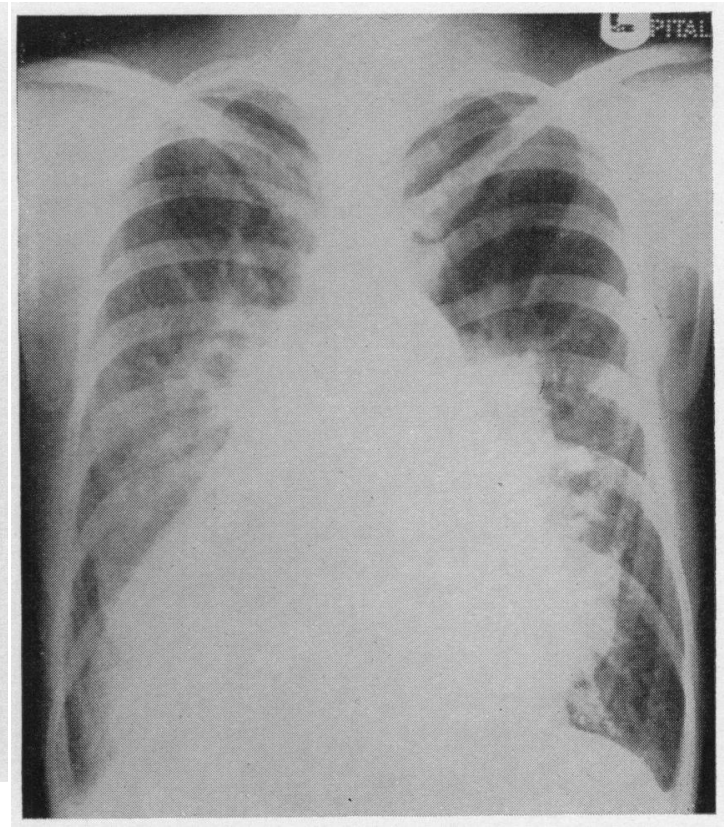

FIG. 4.-Single auricle. Dextrocardia. Bi-directional shunt. Severe pulmonary hypertension. There is enormous enlargement of the pulmonary arteries, and the small branches appear well filled out to the periphery.

\section{(c) Congenital Heart Disease without Severe Pulmonary Hypertension}

Examination of this group revealed essentially similar changes to those seen in the hypertensive group in that all had enlargement of the main and medium-sized arteries. Two patients, however, had oligæmia of the periphery of the lung, similar to that seen in the hypertensive group, and in one patient obvious costophrenic lines were seen.

While oligæmia at the periphery of the lung usually indicated reduction in, or reversal of, the left-to-right shunt due to increased vascular resistance, this was not always the case, and it was 


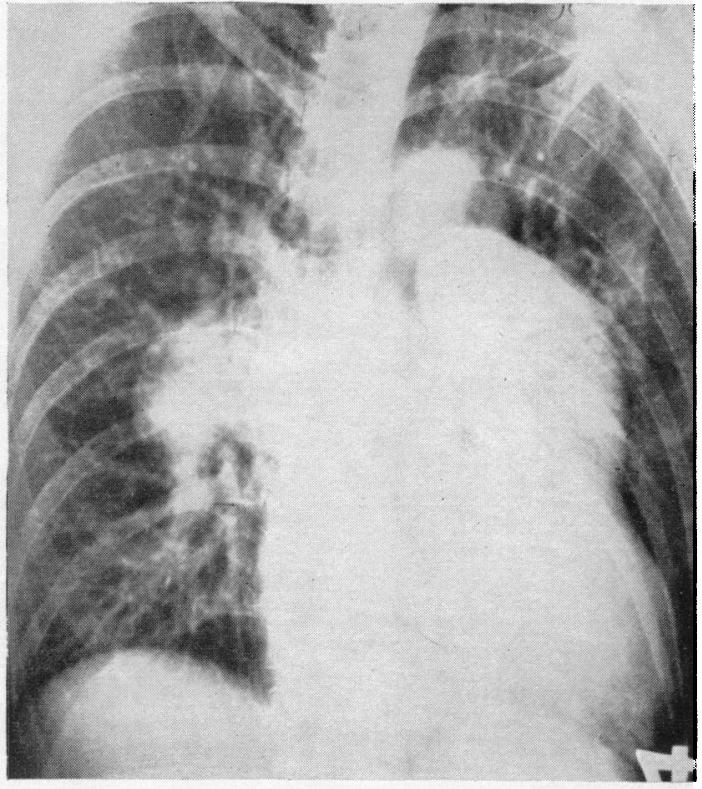

FIG. 5.-Angiocardiogram of ventricular septal'defect with severe pulmonary hypertension and right-tolefti shunt, showing great enlargement of the large pulmonary arteries, but deficient filling and narrowing of the peripheral branches in both upper and lower zones. Early filling of the aorta from the right ventricle can be seen.

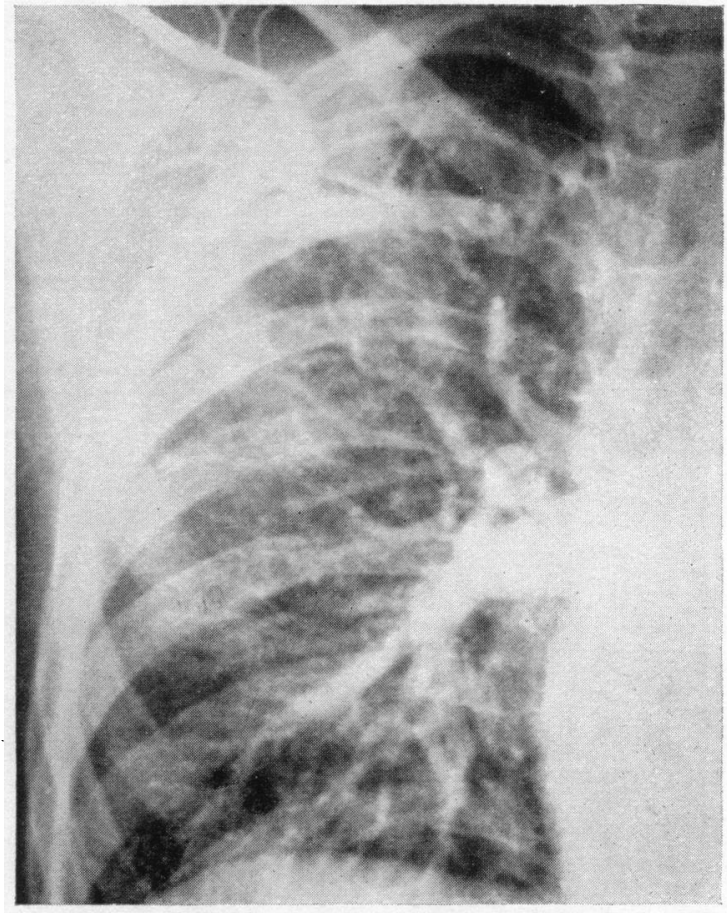

FIG. 6.-Angiocardiogram of ventricular septal defect with severe pulmonary hypertension and right-toleft shunt. The large pulmonary arteries are greatly enlarged, but the small branches are of normal size. No peripheral oligæmia.

not possible to determine the presence or absence of severe pulmonary hypertension on examination of the chest film except when the hypertension was very severe.

Fig. 3 shows the chest film of a patient with an atrial septal defect. Her systolic pulmonary arterial pressure was $90 \mathrm{~mm} . \mathrm{Hg}$, and there is great enlargement of the main and intermediary branches and peripheral oligæmia. By contrast, Fig. 4 shows the chest film of a patient with dextrocardia and single atrium, whose pulmonary artery systolic pressure was identical, yet no peripheral oligæmia can be seen. In Fig. 7, however, there is peripheral underfilling in a child with an atrial septal defect whose pulmonary artery systolic pressure was normal, and the left-toright shunt large. Fig. 5 and 6 contrast the appearances in two patients with severe pulmonary hypertension.

\section{The Effects of Hypotensive Agents upon the Pulmonary Circulation}

(a) Mitral Stenosis. We have found (Davies et al., 1954) that hexamethonium can reduce the pulmonary arterial pressure in severely hypertensive patients without a significant fall in cardiac output or systemic arterial pressure, suggesting a specific effect upon the pulmonary vasculature. We have recently extended these studies, and have found that the fall in pulmonary vascular resistance may be greater than the systemic, even in cases with mild pulmonary hypertension. These results will be reported fully later.

(b) Congenital Heart Disease. Seven patients with shunts and severe pulmonary hypertension have been given hexamethonium or tolazoline during cardiac catheterization. Six had bi-directional 


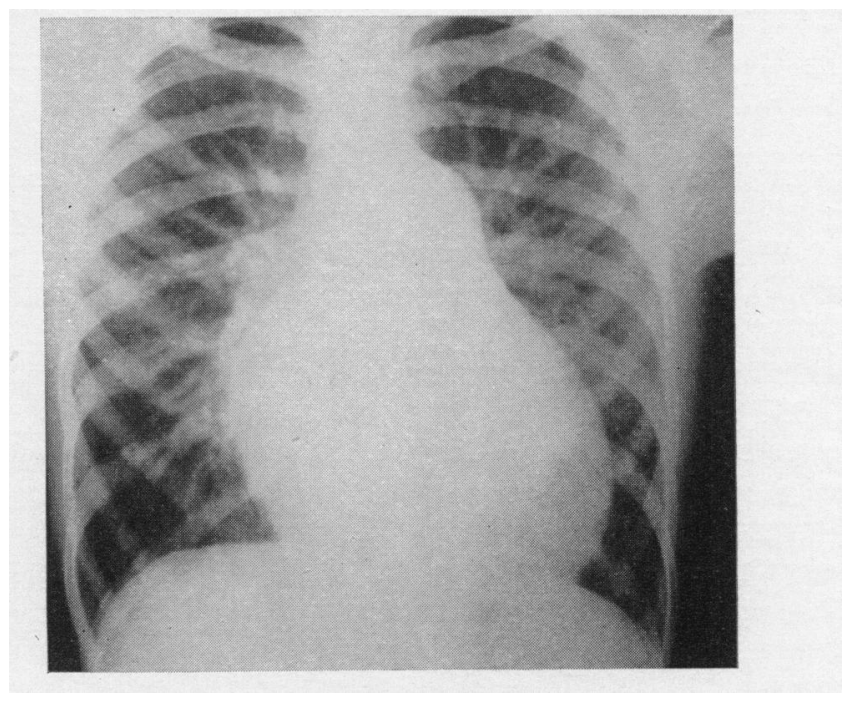

Fig. 7.-Atrial septal defect, but no pulmonary hypertension. The main pulmonary arteries are enlarged, but the medium sized branches are normal, and there is apparent underfilling of the small branches producing peripheral oligæmia.

shunts with severe pulmonary hypertension, and one had severe pulmonary hypertension, but no right-to-left shunt.

In three patients there was no fall in pressure, in two it was slight, and in the remaining one, moderate (but associated with a comparable fall in cardiac output).

The ready fall obtained in cases of mitral stenosis with comparable levels of pulmonary hypertension was never seen. The results of this study are not yet complete and will be reported in full elsewhere.

\section{(a) Mitral Stenosis}

\section{POST-MORTEM PUlmonARY ARTERIOGRAPHY}

Arteriograms technically suitable for assessment were available in 30 cases. The pulmonary artery pressure had been measured in life in 15 and the systolic pressure was greater than $70 \mathrm{~mm}$. $\mathrm{Hg}$ in 8 of them.

Plain films during life had shown pulmonary hypertensive changes in 19 cases; the remaining films could not be assessed for various reasons such as the presence of hæmosiderosis, pulmonary œdema, or infarction.

In assessing post-mortem arteriograms certain arbitrary divisions of position and size were made. It was found that different groups of segments had different appearances and that the grouping did not correspond with the anatomical lobes. Thus, the middle lobe or lingular arteries behaved like lower lobe ones and arteries of the apical segment of the lower lobe behaved like upper ones. We shall, therefore, refer to upper and lower zone arteries and not to the lobes. Also, for convenience we shall refer to arteries of the following groups of size: main pulmonary arteries, segmental arteries and their primary divisions, small arteries (down to about $0.5 \mathrm{~mm}$. lumen diameter), and background (arteries too fine to be seen clearly individually). In assessing the size of the pulmonary arteries, each case was compared with controls of similar age and lung size.

The following changes were noted in the arteriograms.

Main pulmonary arteries. In 13 cases the main right or left pulmonary artery was enlarged, but in 17 cases there was no significant difference from the controls. 
Segmental arteries and their primary branches. In this group the upper and lower zone arteries were different in appearance and they will therefore be considered separately. The upper zone arteries were enlarged in 11 cases and some of these were slightly tortuous; they were of normal size in 18 cases and slightly narrowed in one case. The lower zone arteries on the other hand were enlarged only in 2 cases, were of normal size in 14, narrowed in 12, and could not be assessed owing to emboli in 2 cases. In this group of arteries, those of the upper zone were usually enlarged or normal while those of the lower zone were normal or narrowed, consequently there was a difference in the size of the arteries of the two zones, the upper being the larger, in 19 cases.

Small arteries. These showed much the same changes as the larger branches but with a greater zonal difference. In the case of the upper zone arteries, 10 cases showed enlargement, 16 were of normal size, and 4 were narrowed. In the lower zone arteries one case only showed enlargement, seven were of normal size, and 19 were narrowed; three could not be assessed owing to multiple emboli. Of the 27 cases that could be assessed, 23 showed a difference in arterial calibre between the two zones. Background filling was diminished in 22 cases and normal in 8 . In 16 of the 22 cases with diminished filling the lower zone was less well filled than the upper.

The vascular narrowing so far considered has always been a regular narrowing affecting all of a group of vessels equally. In some cases, however, a different type of focal narrowing was seen. It affected individual arteries, nearly always in the group of segmental or their first divisions, irregularly along their length and to a very severe degree. In all such cases only arteries of the lower zone were affected. This type of narrowing was proved to be due to atheroma and generally of great severity. It was seen in 12 out of the 30 cases and it was closely correlated with the presence of thrombotic or embolic arterial occlusion. Ten cases showed thrombo-embolic occlusion and in 8 of these there was focal narrowing in the other arteries.

Fig. 8 shows an arteriogram of a case of mitral stenosis paired with a control of similar age and lung size for comparison. There is striking narrowing of the primary segmental, medium, and small branches confined to the lower zones.

\section{(b) Congenital Group}

In this group 4 cases of atrial septal defect, one case of single atrium, and one case of patent ductus arteriosus were available for post-mortem examination. In four of these full investigation with angiography was carried out; in the other two the lungs were obtained after a necropsy elsewhere and only ordinary dissection and histology were possible. Since the findings in these two cases were the same as in the four injected cases we feel justified in including them. In the four cases in which the systolic pulmonary artery pressure was measured it was grossly raised $(70-128 \mathrm{~mm} . \mathrm{Hg})$, and in life all had shown marked dilatation of the main pulmonary arteries, and major branches, with reduction in size of the fine peripheral arteries. This reduction was equally distributed throughout the lung, so that there was oligæmia of the peripheral lung fields in all zones. In one of the cases examined by dissection only, the systolic pulmonary artery pressure had been $90 \mathrm{~mm} . \mathrm{Hg}$ and in the other $40 \mathrm{~mm}$. $\mathrm{Hg}$.

In all 6 cases the main pulmonary arteries were dilated, moderately in four and enormously in two. The segmental arteries and their primary branches were dilated in all cases. This was enormous in three and in the other three as great as in any of the mitral cases. In all there was accompanying tortuosity. The dilatation was equally severe in the upper and the lower zones and unlike the mitral groups, no difference could be detected.

The smaller arteries could only be estimated in the four injected cases. In all these the second divisions of the segmental arteries were a little enlarged but with further divisions the arteries very rapidly diminished in size so that most small arteries were narrowed. The background filling was minimal in all cases. As in the case of the larger arteries and in contrast to the mitral cases there was no difference between the upper and lower zones. 


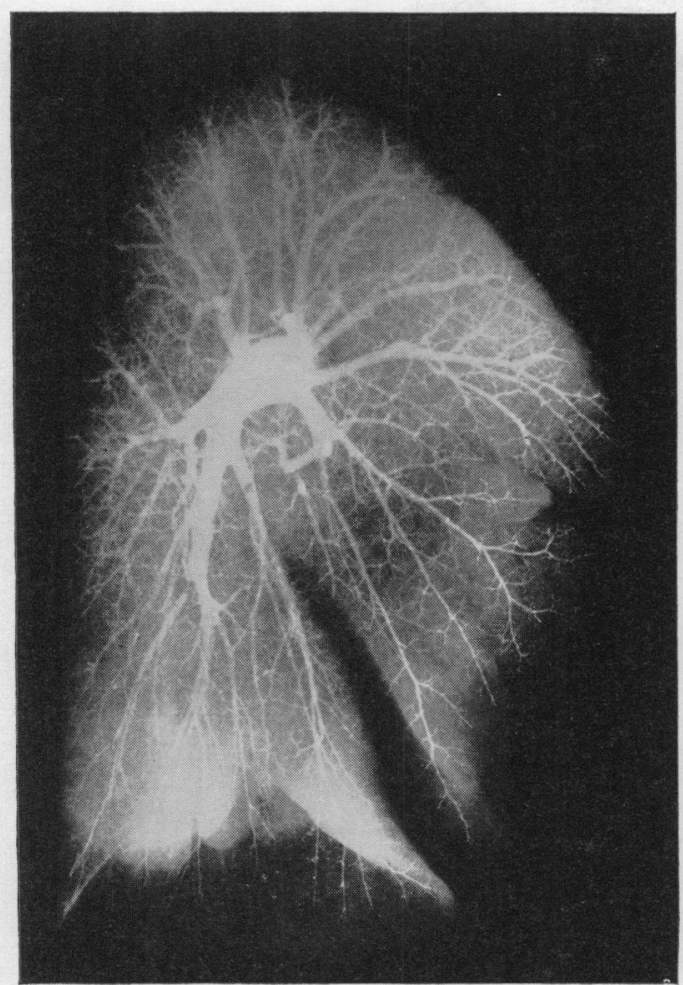

MS

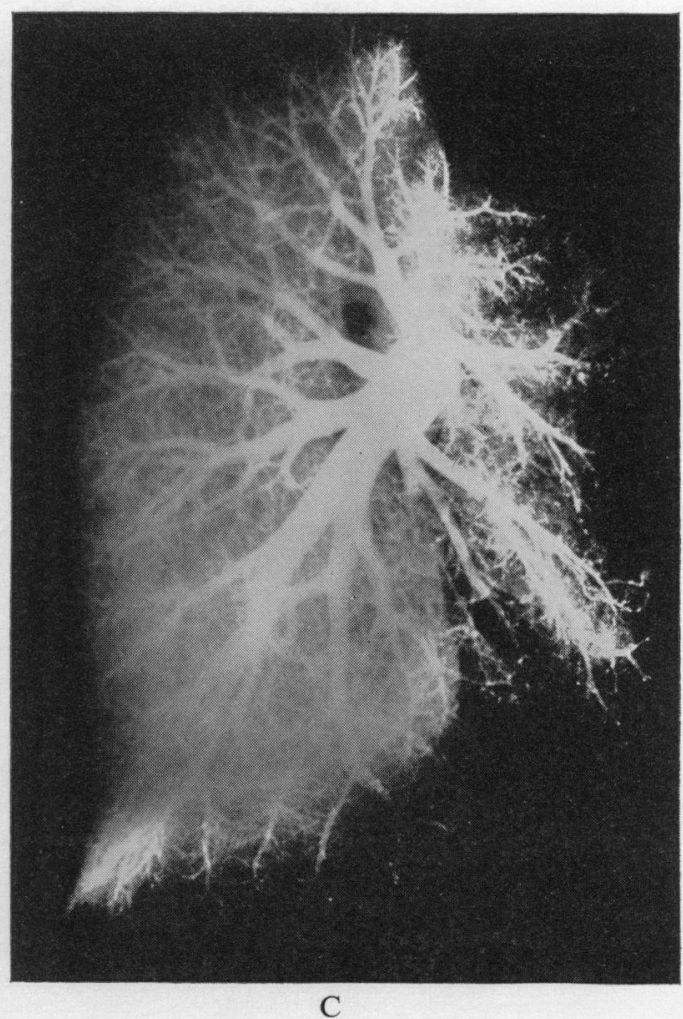

$\mathrm{C}$

FIG. 8.-Post-mortem pulmonary arteriogram in a case of mitral stenosis (MS), compared with a control (C). There is narrowing of the primary segmental, medium, and small-sized pulmonary arteries confined to the lower zones.

None of these cases showed atheromatous narrowing, either in the arteriograms or on subsequent dissection. Fig. 9 shows the appearances in a patient with an atrial septal defect who had severe pulmonary hypertension in life, compared with a control of similar age and lung size. There is great tortuosity and enlargement of the pulmonary arteries extending down to the mediumsized branches, but there is striking reduction in " background " filling which is distributed equally throughout all zones of the lung (see also Fig. 3).

\section{Macroscopical Dissection}

(a) Mitral Stenosis. The changes in vascular size noted in the radiographs were confirmed. The two types of narrowing, focal and diffuse, were found to correspond to two different processes. The ordinary diffuse narrowing seen in the lower zones was due to uniform thickening of the artery, apparently by contraction. The focal narrowing was due to intimal plaques of atherosclerosis bulging into the lumen and narrowing it locally. Minor degrees of atherosclerosis were present in most cases but these did not encroach on the lumen sufficiently for any changes to be detectable in the radiographs or on dissection. Thrombotic or embolic occlusions with corresponding infarcts, old or recent, were present in 10 cases.

A feature that became apparent on dissection of the injected and uninjected lungs was the curious distribution of congestion and œdema. These both showed a tendency to localize in the central and upper parts of the lungs. In the ordinary case with death in congestive failure this distribution was discernible but not obvious. It was obvious in cases of acute cardiac failure with 


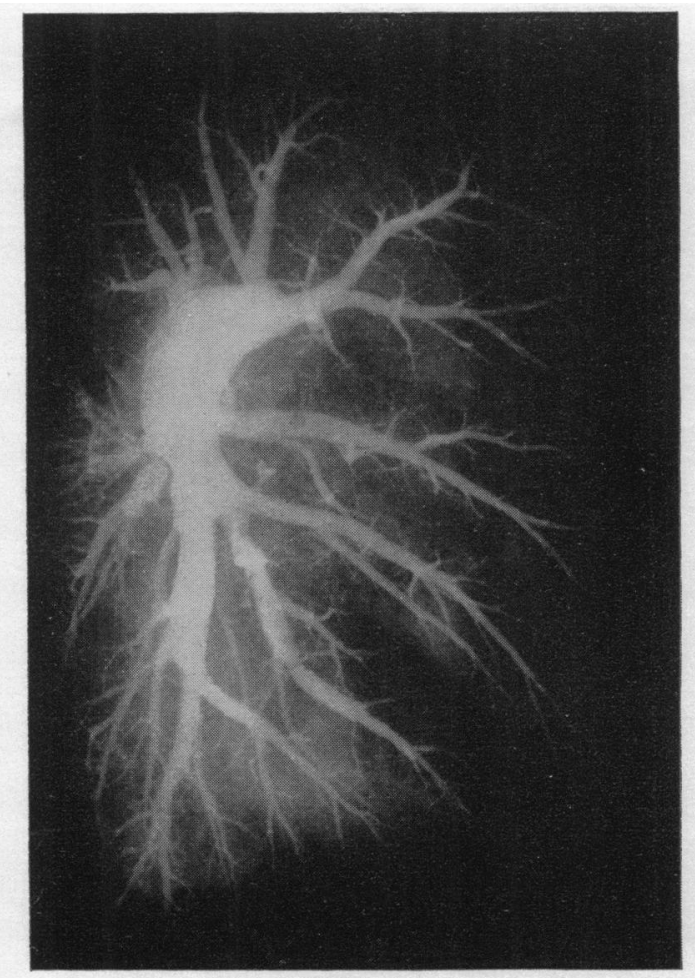

(A.S.D.)

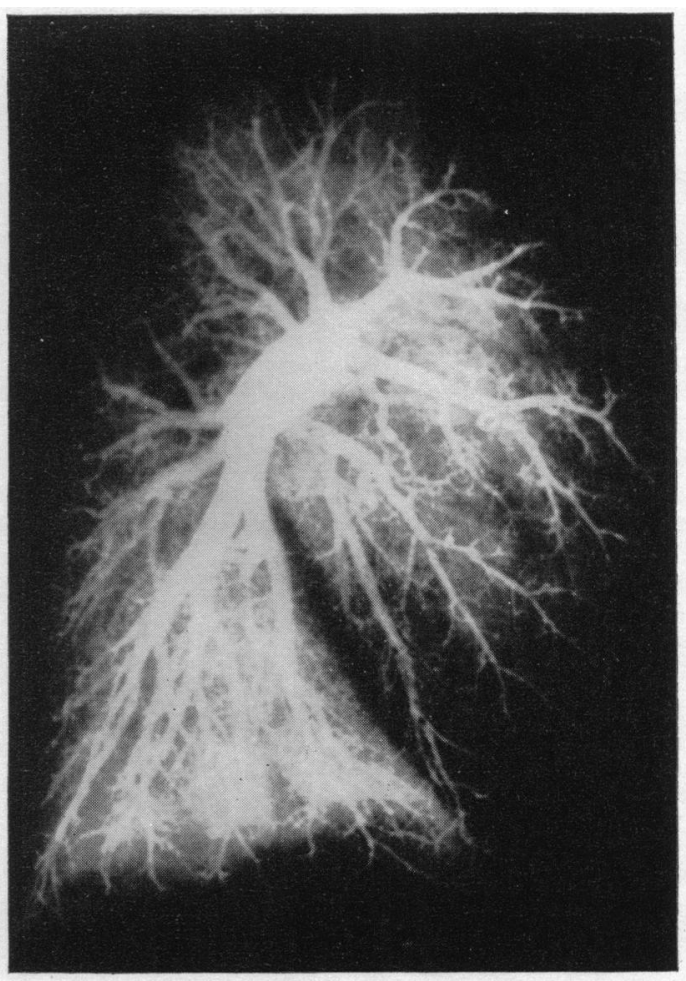

(C)

FIG. 9.-Post-mortem pulmonary arteriogram in atrial septal defect (ASD) with severe pulmonary hypertension, compared with a normal control (C) of similar age and lung size. There is great enlargement and tortuosity of the pulmonary arteries extending down to the small branches, but there is loss of background filling due to involvement of the very small arteries, which is equally distributed in upper and lower zones.

pulmonary œdema. In such cases there was extreme congestion of the central and upper zones with œdema and little of either in the lower zones (Fig. 10). This peculiar distribution has been described by Jackson (1951) and was also noted in uræmia by Doniach (1947).

(b) Congenital Group. There was no evidence of atheromatous narrowing on dissection of the pulmonary arterial tree.

\section{Histology}

(a) Mitral Stenosis. Two processes were seen in the arteries, hypertrophy and atherosclerosis. The former was seen at all levels. It could only be estimated with certainty by comparing the thickness of the vessel wall with that of an exactly corresponding artery from a control lung. The degree of hypertrophy varied between the upper and lower zones, the latter always being the greater. In some cases this difference was of minor degree, in others it was considerable (Fig. 11A). This hypertrophy affected only the media and was totally independent of any intimal thickening that was present. Intimal thickening occurred as atherosclerosis to some degree in most cases. It showed exactly the same distribution as the hypertrophy, affecting the lower zones. In the majority of cases it took the form of fatty infiltration of a slightly thickened intima and affected only segmental arteries or their primary divisions. Such intimal thickening had no effect on the lumen and was comparable with the superficial fatty streaking of the aorta of young people. Severe atheroma, of the type seen in peripheral arteries in the greater circulation, occurred in a minority. 


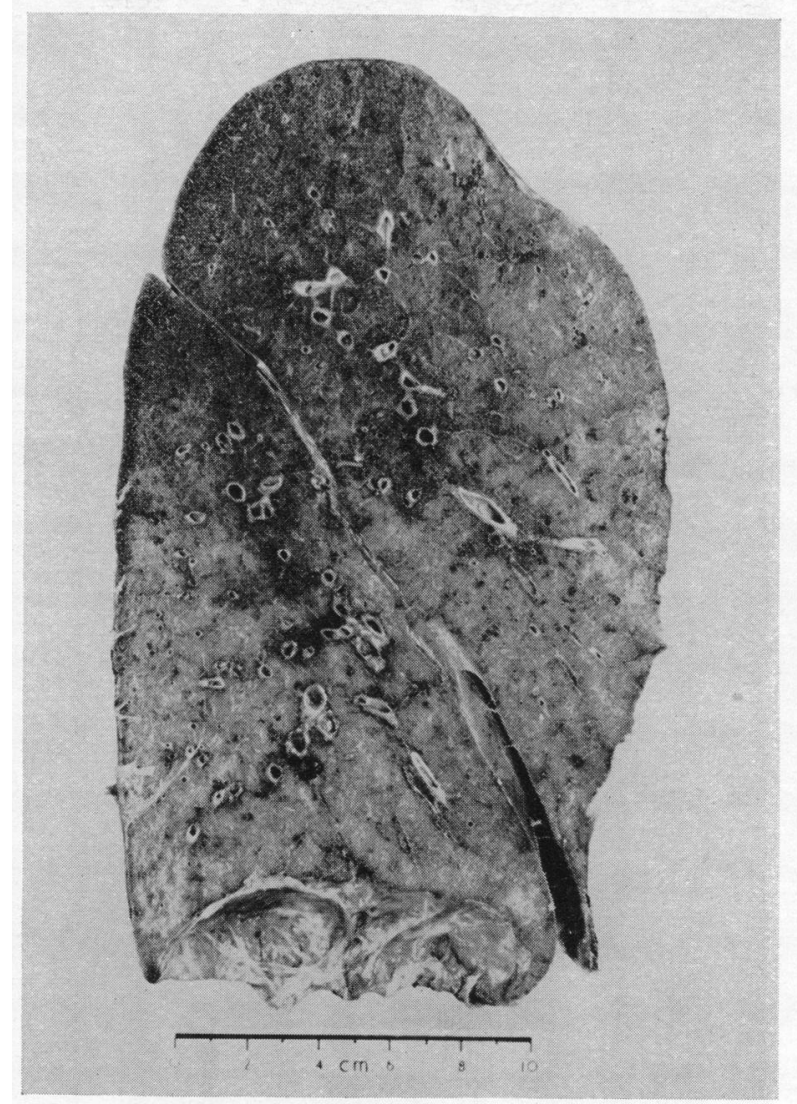

FIG. 10.-Photograph of left lung in patient with mitral stenosis and severe pulmonary hypertension who died in pulmonary cdema. The congestive changes are confined to the central and upper zones, and are absent from the bases.

In 13 out of 30 cases there was some such atherosclerosis but in many of these only a few arteries were affected. In every case in which atherosclerosis was severe enough to affect the lumen of a pulmonary artery it occurred in a lower zone artery. Most of such narrowing was focal and almost invariably the narrowing seen in the lower zones in angiograms was due to the muscular contraction of hypertrophied arteries and was only very rarely due to irreversible atherosclerosis.

The pulmonary veins showed hypertrophy analogous to that seen in the arteries and in them too hypertrophy was greater in the lower zones (Fig. 11B).

(b) Congenital Group. In spite of their dilatation, the proximal arteries showed hypertrophy. The distal arteries, where they were of similar diameter to the controls, also showed hypertrophy. The degree of medial hypertrophy was of the order of 2 to 3 times both centrally and peripherally and in contrast to the mitral cases there was no detectable difference in the degree of hypertrophy in the upper and lower zones (Fig. 11c). Atherosclerosis was minimal in all cases but the number examined was too small for this finding to be of much significance. Changes of the type described by Brewer (1955) were noted but these affected only the smallest arteries and are not germane to the present problem. 


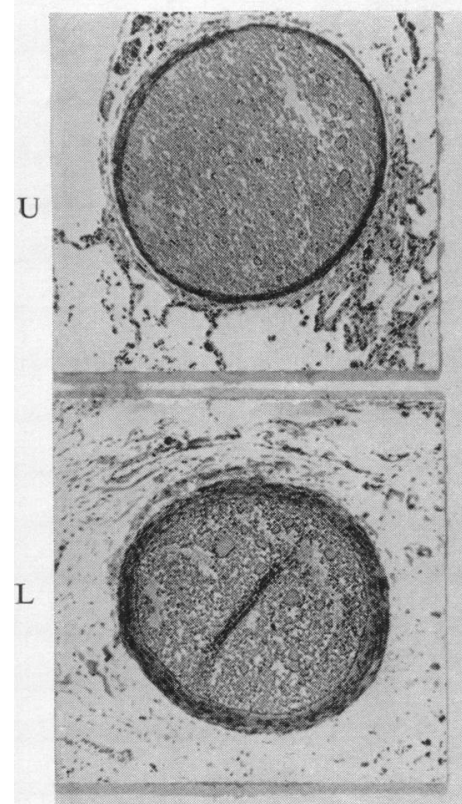

A
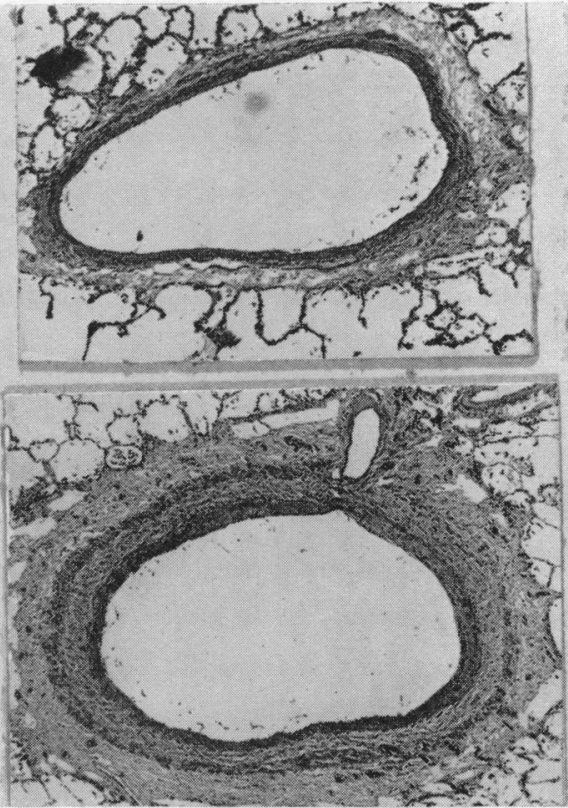

B

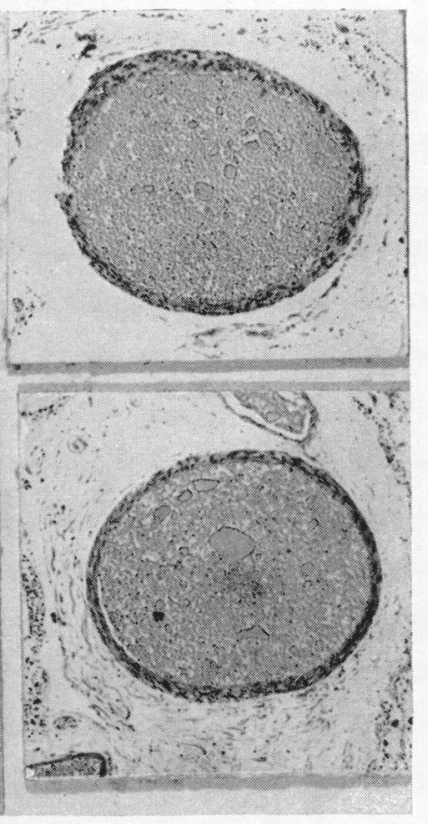

C

FIG. 11.-(A) Small pulmonary arteries from the lower (L) and upper (U) lobes in a patient with mitral stenosis (the same case as Fig. 8). The vessels are of comparable size $(2 \mathrm{~cm}$. from pleura), and of equal magnification. Injection mass fills the lumen of each vessel. The thickness of the media in the lower lobe vessel is 2 to 3 times that of the upper. $\times 45$. (B) Pulmonary veins from the lower (L) and upper (U) lobes in a patient with mitral stenosis (same case as Fig. 8). The vessels are of comparable size $(2 \mathrm{~cm}$. from pleura), and equal magnification. The thickness of the media of the lower lobe vein is 4 times that of the upper. $\times 24$. (C) Small pulmonary arteries from the lower (L) and upper (U) lobes in a case of atrial septal defect with severe pulmonary hypertension. The vessels are of comparable size $(2 \mathrm{~cm}$. from pleura), and equal magnification. Injection mass fills the lumen. The thickness of the media is equal in both vessels and is 2 to 3 times that of normal. $\times 45$.

\section{Discussion AND Conclusions}

These studies show that there are differences in the pulmonary vascular patterns and behaviour between mitral stenosis and congenital heart disease with shunts. These differences are most striking (especially in mitral stenosis) when severe pulmonary hypertension is present.

In mitral stenosis, as we have previously shown (Davies et al., 1953), the degree of narrowing of the peripheral arteries and of enlargement of the main arteries is proportional to the severity of the pulmonary hypertension present. The narrowing of the small branches was confined to the lower and mid zones of the lungs and did not affect the apical regions; the narrowing involved arteries as proximal as branches of the segmental arteries. These changes can be seen in angiocardiograms in life, and in many cases in plain X-rays. They were confirmed by post-mortem arteriography and histological studies (Fig. 1, 2, 8, and 11).

In congenital heart disease there was considerable enlargement, dilatation, and tortuosity of the main and major branches of the pulmonary artery when a left-to-right shunt was present. This enlargement often extended into the small branches, which were also tortuous. Post-mortem arteriography has shown that when severe pulmonary hypertension has been present in life, there is narrowing of the very small branches which shows as lack of " background " filling, and that even small branches may be dilated and tortuous. This loss of " background " filling is uniform throughout the lung and there is no localization to the lower zones, in sharp contrast to mitral stenosis (Fig. 9). Histological studies confirm these differences in distribution (Fig. 11c). 
Radiological studies in life, however, do not give a clear indication of the presence or absence of severe pulmonary hypertension in the congenital group, again in contrast to mitral stenosis, and while oligæmia of the peripheral lung fields may be seen in severe hypertensive cases with bidirectional shunts (Fig. 3 and 5), there are many exceptions (Fig. 4 and 6). It is probably safe to say, however, that its presence usually indicates severe hypertension and actual or impending reversal of the left-to-right shunt.

Since the narrowing of the pulmonary arteries involves the smallest branches it is not surprising that it is not detectable in plain X-rays or even on angiography (Fig. 6).

It is probable that high pulmonary arterial pressures in this group can result from increases in flow, which dilate even the small arteries. In cases with a large left-to-right shunt and torrential pulmonary blood flow this dilatation then extends into branches at the periphery of the lungs, giving a picture of overfilled vessels in the presence of a high pulmonary pressure. When the resistance rises in the very small vessels, the left-to-right shunt tends to be reduced, and thus the dilatation of the peripheral vessels becomes less. Therefore the state of the peripheral pulmonary arteries, and the degree of filling seen radiologically will depend upon the balance between the magnitude of the left-to-right shunt and the resistance offered by the smallest arteries and arterioles.

In mitral stenosis the situation is less complex, since the pulmonary blood flow is not increased and the small branches of the pulmonary artery are not dilated as a result. Dilatation of the main pulmonary arteries is the result of pulmonary hypertension alone. Furthermore, the localization of the arterial narrowing to the lower zones, and its presence in branches of a size easily detectable radiologically, permits a reliable correlation between pressure and radiological appearances.

A further contrast between the two groups, which is of great interest although not necessarily directly related to the differences in vascular patterns, lies in the response to hypotensive drugs. The ready fall in pulmonary vascular resistance obtained in mitral stenosis did not occur in the congenital group. The latter group, however, are extremely difficult to study in this way; the results of further work will be reported elsewhere.

The Genesis of the Different Vascular Patterns in Mitral Stenosis and Congenital Heart Disease. We believe that the clue to the difference between the two groups lies in the pulmonary venous pressure. This is not uniform throughout the lungs because of hydrostatic differences between different areas. In the erect posture it will be higher at the bases than the apices.

In mitral stenosis, the left atrial pressure is significantly increased by the obstruction of the mitral valve. This results in pulmonary venous hypertension which will be augmented at the bases by the hydrostatic increment, and this may reach a critical level for oedema formation. We believe that this critical venous pressure results in arterial constriction in the lower, and later in the mid zones. Because of the negative hydrostatic pressure at the apices relative to the left atrium, very high left atrial pressures, well above the critical level for œdema formation, would be required for arterial constriction to occur at the apices; we have never seen this. The finding of greater muscular hypertrophy in lower lobe pulmonary veins than in upper lobe veins strongly supports this explanation (Fig. 11B).

It has been suggested that in mitral stenosis the pulmonary arterial pressure rises passively in proportion to the left atrial pressure, until the latter reaches a critical level for œdema formation, after which small increments of left atrial pressure cause large increases in pulmonary arterial pressure indicating active arteriolar constriction (Dexter et al., 1950; Holling, 1952; Blacket et al., 1953).

We believe, however, that active arterial constriction at the bases of the lung may develop at lower levels of left atrial pressure. In a previous report (Davies et al., 1954) it was shown that in patients with severe pulmonary hypertension the injection of small amounts of hexamethonium into the pulmonary artery is followed by a larger fall in pulmonary, than in systemic, arterial pressure, without a fall in cardiac output. Our more recent observations have shown a similar selective fall in pulmonary vascular resistance in patients with only modest pulmonary hypertension. These results will be reported elsewhere. 
The radiological appearances of diffuse haziness in the lower zones probably represents chronic transudation from the capillaries which is the result of the locally increased basal venous pressure. By contrast " acute massive" pulmonary œdema is diverted to the central core of the lung, and sometimes to the apices, by the arterial vasoconstriction at the bases which prevents extensive rapid transudation there. Fig. 10 shows pulmonary œdema confined to the mid and apicoposterior zones in a patient who had severe mitral stenosis with pulmonary hypertension in life. The localization of the transverse costophrenic lines to the bases harmonizes with this concept and suggests that they are due to interlobular œdema, as suggested by Fleischner and Reiner (1954), although impaired lymphatic drainage (Shanks and Kerley, 1951), lymphatic dilatation (Levin, 1955), or over production of lymph may be a factor.

Our findings shed some light on the concept of a " protective" increase in arteriolar resistance which has been said to prevent pulmonary œdema (Lewis et al., 1952; Logan and Turner, 1953; Wood, 1954). We have previously challenged this concept (Davies et al., 1954) believing that patients with severe pulmonary hypertension and arterial disease are as likely, or more likely, to have attacks of œdema as those with modest pulmonary arterial hypertension and considerable pulmonary venous hypertension. The results of the present investigation indicate that the arterial changes prevent massive pulmonary œdema only at the bases, and divert it to other portions of the lung (upper and central zones). It would thus be reasonable to speak of " redistributive ", or perhaps "locally protective" arterial narrowing.

In congenital heart disease with pulmonary hypertension and a shunt, the pulmonary venous pressure is usually normal (Swan et al., 1954; Bruwer et al., 1955; Rossall and Gunning, 1956). Therefore, the hydrostatic increment that exists at the bases will not increase the venous pressure there to a critical degree. A critically raised pulmonary venous pressure can, therefore, be excluded as a cause of the arterial changes in the congenital group, so that in contrast to mitral stenosis, the arterial changes are generalized throughout the lungs.

It has previously been suggested that narrowing of the pulmonary arterioles is, at least in part, due to the torrential pulmonary flow (Hultgren et al., 1953; Civin and Edwards, 1950), and our findings of narrowing in the small arteries in all zones of the lungs are consistent with this hypothesis, although we can offer no proof.

However, other factors may be implicated, such as abnormalities of the vessels themselves (Civin and Edwards, 1951; Welch and Kinney, 1948; Evans, 1951).

The extreme rarity of acute or chronic pulmonary œdema in congenital heart disease with shunts is likely to be due to the low levels of left atrial pressure. However, horizontal costophrenic lines are sometimes seen, although not as frequently or extensively as in mitral stenosis (Bruwer et al., 1955; Rossall and Gunning, 1956). They were present in 4 of our congenital cases (3 with severe pulmonary hypertension). We believe that they are then the result of either increased left atrial pressure due to torrential left atrial flow, or left ventricular failure, or perhaps to abnormalities of lymph flow induced by the large pulmonary blood flow.

\section{SUMMARY}

A combined radiological, hæmodynamic, and pathological study of the pulmonary vascular system has been made in two groups of cases-mitral stenosis and congenital heart disease with pulmonary hypertension and shunts.

Striking differences in the pulmonary vascular pattern were found between the two groups.

In mitral stenosis, arterial narrowing was confined to the lower and mid zones, and involved the branches of segmental arteries, but was not present in the upper zones.

In the congenital group all the branches were dilated and tortuous, except the very fine arteries which were reduced in size. There was no regional localization; the changes being diffuse throughout the upper, mid, and lower zones.

There were also differences between the two groups in the response of the pulmonary vasculature to hypotensive drugs. The reasons for the differences are discussed. 
We are grateful to Dr. J. D. K. North and Dr. G. H. Neilson for help with certain aspects of this work. We also wish to thank Professor J. McMichael for help and advice; Mr. Brecknell, and the staff of the Department of Photography for preparing the illustrations.

\section{REFERENCES}

Blacket, R. B., Palmer, A. J., Sinclair-Smith, B. C., Farrar, J. F., Halliday, J. H., and Maddox, J. K. (1953). Aust. Ann. Med., 2, 36.

Brewer, D. B. (1955). J. Path. Bact., 70, 299.

Bruwer, A. J., Ellis, F. H., Jr., and Kirklin, J. W. (1955). Circulation, 12, 807.

Civin, W. H., and Edwards, J. E. (1950). Circulation, 2, 545.

- - (1951). Arch. Path., 51, 192.

Davies, L. G., Goodwin, J. F., Steiner, R. E., and Van Leuven, B. D. (1953). Brit. Heart J., 15, 393.

,-- , and Van Leuven, B. D. (1954). Brit. Heart J., 16, 440.

Dexter, L., Dow, J. W., Haynes, F. W., Whittenberger, J. L., Ferris, B. G., Goodale, W. T., and Hellems, H. K. (1950). J. clin. Invest., 29, 602.

Doniach, I. (1947). Amer. J. Roentgenol., 58, 620.

Evans, W. (1951). Proc. Royal Soc. Med., 44, 600.

Fleischner, F. G., and Reiner, L. (1954). New Engl. J. Med., 250, 900.

Goodwin, J. F., Steiner, R. E., and Lowe, K. G. (1952). J. Fac. Radiol., Lond., 4, 21.

Harrison, W., and Wood, P. (1949). Brit. Heart J., 11, 205.

Hultgren, H., Selzer, A., Purdy, A., Holman, M., and Gerbode, F. (1953). Circulation, 8, 15.

Holling, H. E. (1952). Brit. med. Bull., 8, 358.

Jackson, F. (1951). Brit. Heart J., 13, 503.

Levin, B. (1955). Amer. Heart J., 49, 521.

Lewis, B. M., Gorlin, R., Houssay, H. E. J., Haynes, F. W., and Dexter, L. (1952). Amer. Heart J., 43, 2.

Logan, A., and Turner, R. (1953). Lancet, 1, 1007.

Rossall, R. E., and Gunning, A. J. (1956). Lancet, 1, 604.

Shanks, S. C., and Kerley, P. (ed.) (1951). Text Book of X-ray Diagnosis by British Authors. 2nd ed., Vol. 2. London: H. K. Lewis.

Swan, H. J. C., Zapata-Diaz, J., Burchell, H. B., and Wood, E. H. (1954). Amer. J. Med., 16, 12.

Welch, K. J., and Kinney, T. D. (1948). Amer. J. Path., 24, 729.

Wood, P. (1954). Brit. med. J., 1, 1051 and 1113. 